\title{
The asymptotic distribution of the number of summands in unrestricted $\Lambda$-partitions
}

by

\author{
D. V. LEE (Nottingham)
}

By an unrestricted $\Lambda$-partition of $n$ we mean a solution in non-negative integers $a_{i}$ of

$$
\sum_{i=1}^{\infty} a_{i} \lambda_{i}=n,
$$

where $\Lambda=\left\{\lambda_{1}, \lambda_{2}, \ldots\right\}$ is an infinite multiset of positive integers. Much work has been done, see for example [5], on statistical aspects of the restricted partitions with $a_{i} \leq 1$, but little appears to be known about the distribution of the number of summands in the solutions of (1) in the unrestricted case beyond the theorem of Erdös and Lehner for the case $\Lambda=\mathbb{N}$, which shows in particular that if all solutions of (1) are equally likely, then the random variable

$$
\pi\left(\frac{2}{3}\right)^{1 / 2} \sum_{i=1}^{\infty} a_{i} / n^{1 / 2} \log n
$$

converges in distribution to unity as $n \rightarrow \infty$. For further details, see [4].

In the paper of Loxton and Yeung [7], there is the following question communicated to the authors by Erdös: "Does there exist $f(c)$ such that the number of partitions of $n$ into squares in which the number of summands is less than $c n^{2 / 3} \log n$ is asymptotic to $f(c) p_{2}(n)$ ?" Here $p_{2}(n)$ is the total number of partitions of $n$ into squares.

As we shall see, the answer to this problem is

$$
f(c)= \begin{cases}0, & c=0, \\ 1, & c>0,\end{cases}
$$

and a more appropriate question is obtained by replacing $c n^{2 / 3} \log n$ by $c n^{2 / 3}$, when the corresponding $f(c)$ is continuous. We shall derive the equivalent of $f(c)$ for a wide range of sets $\Lambda$, including $\mathbb{N}$ and the set of squares.

We denote by $P_{\Lambda}(n)$ the number of solutions of (1), and let $\Lambda_{k}$ be the multiset consisting of $\Lambda$ together with $k$ copies of unity. As a prerequisite 
for the proof of our theorem, we must have asymptotic formulae for $P_{\Lambda}(n)$ and $P_{\Lambda_{k}}(n)$ of a particular form; these are shown to exist, with certain restrictions on $\Lambda$, by Meinardus in [8] and by Ingham, Auluck and Haselgrove in [6] and [2]. The former result can also be found in Chapter 6 of [1]. We state a suitable version of the latter result. If

TheOREM (Ingham, Auluck, Haselgrove). Let $\Lambda$ be a multiset as above.

$$
N(n)=\sum_{\substack{i \\ \lambda_{i} \leq n}} 1
$$

satisfies

$$
N(n)=A \alpha^{-1} n^{\alpha}+R(n),
$$

where $\alpha, A>0$ and, as $u \rightarrow \infty$,

$$
\int_{0}^{u} \frac{R(v)}{v} d v=a \log u+b+o(1),
$$

and the elements of $\Lambda$ have no non-trivial common factor, then

$$
\begin{aligned}
P_{\Lambda}(n) \sim\{2 \pi(1+\alpha)\}^{-1 / 2} e^{b} M^{-(a-1 / 2)} n^{-1 / 2+(a-1 / 2) /(\alpha+1)} \\
\times \exp \left\{(1+1 / \alpha) M n^{\alpha /(\alpha+1)}\right\}
\end{aligned}
$$

as $n \rightarrow \infty$, where

$$
M=\{A \Gamma(\alpha+1) \zeta(\alpha+1)\}^{1 /(\alpha+1)} .
$$

If Meinardus's theorem applies also, then the quantities $A$ and $\alpha$ above are equal to Meinardus's $A$ and $\alpha$. Now we can state our theorem.

Theorem. Suppose $\Lambda=\left\{\lambda_{1}, \lambda_{2}, \ldots\right\}, \lambda_{1} \leq \lambda_{2} \leq \ldots$, satisfies the conditions of the theorem of Ingham et al. (or the conditions of Meinardus's theorem); suppose also that $1 \in \Lambda$ or there is no $\lambda_{i}$ such that $\Lambda \backslash\left\{\lambda_{i}\right\}$ has all its elements divisible by a non-trivial common factor. Then if $\alpha=1$ (so that necessarily $\sum \lambda_{i}^{-1}$ diverges), we have

$$
\sqrt{\frac{A \pi^{2}}{6 n}}\left\{\sum_{\substack{i \\ \lambda_{i} \leq n^{1 / 2}}} 1 / \lambda_{i}\right\}^{-1} \sum_{i=1}^{\infty} a_{i}
$$

tends in distribution to one, and if $0<\alpha<1$ (so that $\sum \lambda_{i}^{-1}$ converges), then

$$
M \sum_{i=1}^{\infty} a_{i} / n^{1 /(1+\alpha)}
$$


converges in distribution to the random variable with moment generating function

$$
\prod_{i=1}^{\infty}\left(1-x / \lambda_{i}\right)^{-1}
$$

Hence if the $\lambda_{i}$ are distinct, the limit distribution has distribution function

$$
F(x)=1-\sum_{i=1}^{\infty}\left\{\prod_{\substack{j=1 \\ j \neq i}}^{\infty}\left(1-\lambda_{i} / \lambda_{j}\right)^{-1}\right\} e^{-\lambda_{i} x}, \quad x>0,
$$

provided the sum in (3) converges for $x>0$.

Proof. We consider the auxiliary generating function

$$
g(x)=\prod_{i=1}^{\infty}\left(1-\alpha_{i} x^{\lambda_{i}}\right)^{-1} .
$$

The coefficient of $x_{n}$ in $g(x)$ is

$$
\sum_{\substack{\text { partitions } \\ \Sigma a_{i} \lambda_{i}=n}} \prod_{i=1}^{\infty} \alpha_{i}^{a_{i}}
$$

Let $k$ be a fixed positive integer and let $l_{1}, l_{2}, \ldots$ be non-negative integers with $\sum_{i=1}^{\infty} l_{i}=k$. Let

$$
r^{(l)}=r(r-1) \ldots(r-l+1), \quad l>0, \quad r^{(0)}=1 .
$$

Then

$$
\sum_{\substack{\text { partitions } \\ \Sigma a_{i} \lambda_{i}=n}} \prod_{i=1}^{\infty} a_{i}^{\left(l_{i}\right)}
$$

is the coefficient of $x^{n}$ in

$$
h(x)=\left.\prod_{i=1}^{\infty}\left(\frac{\partial}{\partial \alpha_{i}}\right)^{l_{i}} g(x)\right|_{\alpha_{1}=\alpha_{2}=\ldots=1}=\prod_{i=1}^{\infty} \frac{l_{i} ! x^{l_{i} \lambda_{i}}}{\left(1-x^{\lambda_{i}}\right)^{l_{i}+1}} .
$$

Therefore we have

$$
\sum_{\substack{\text { partitions } \\ \Sigma a_{i} \lambda_{i}=n}} \prod_{i=1}^{\infty} a_{i}^{\left(l_{i}\right)}=\left(\prod_{i=1}^{\infty} l_{i} !\right) P_{\Lambda^{\prime}}\left(n-\sum_{i=1}^{\infty} l_{i} \lambda_{i}\right),
$$

where $\Lambda^{\prime}$ is the multiset obtained from $\Lambda$ by adjoining $l_{i}$ copies of $\lambda_{i}$ for each $i$. We cannot apply the theorems previously mentioned to obtain an asymptotic formula for $P_{\Lambda^{\prime}}(n)$, since $\Lambda^{\prime}$ is not in general independent of $n$. In order to progress, we must restrict the choice of the $l_{i}$ so that $l_{i}=0$ for 
all $i$ such that

$$
\lambda_{i}>n_{0}=n^{1 /(1+\alpha)} / \omega(n),
$$

where $\omega(n) \rightarrow \infty$ slowly; we shall determine the permissible rate of growth of $\omega(n)$ later. We now show that, under this restriction,

$$
P_{\Lambda^{\prime}}\left(n-\sum_{i=1}^{\infty} l_{i} \lambda_{i}\right)=\prod_{i=1}^{\infty} \lambda_{i}^{-l_{i}} P_{\Lambda_{k}}(n)(1+o(1)),
$$

where $o(1)$ depends on the $l_{i}$ only through $k$. Observe that if $\mu_{1}, \ldots, \mu_{k}$ are positive integers and $f(m)$ is a non-decreasing function of $m$, then

$$
\begin{array}{rl}
\prod_{i=1}^{k} \mu_{i}^{-1} \sum_{b_{1}=0}^{\mu_{1}-1} \ldots \sum_{b_{k}=0}^{\mu_{k}-1} & f\left(m-\sum_{i=1}^{k} c_{i} \mu_{i}-\sum_{i=1}^{k} b_{i}\right) \\
\leq & f\left(m-\sum_{i=1}^{k} c_{i} \mu_{i}\right) \\
\leq & \prod_{i=1}^{k} \mu_{i}^{-1} \sum_{b_{1}=0}^{\mu_{1}-1} \ldots \sum_{b_{k}=0}^{\mu_{k}-1} f\left(m-\sum_{i=1}^{k} c_{i} \mu_{i}+\sum_{i=1}^{k} b_{i}\right),
\end{array}
$$

whence if $f(m)=0$ for $m<0$,

$$
\begin{aligned}
\prod_{i=1}^{k} \mu_{i}^{-1} \sum_{\substack{b_{1}, \ldots, b_{k} \\
m-\Sigma b_{i} \geq 0}} f\left(m-\sum_{i=1}^{k} b_{i}\right) \\
\leq \sum_{\substack{c_{1}, \ldots, c_{k} \\
m-\Sigma c_{i} \mu_{i} \geq 0}} f\left(m-\sum_{i=1}^{k} c_{i} \mu_{i}\right) \\
\leq \prod_{i=1}^{k} \mu_{i}^{-1} \sum_{\substack{b_{1}, \ldots, b_{k} \\
m+\Sigma \mu_{i}-\Sigma b_{i} \geq 0}} f\left(m+\sum_{i=1}^{k} \mu_{i}-\sum_{i=1}^{k} b_{i}\right) .
\end{aligned}
$$

By hypothesis and the result of Bateman and Erdős [3], $P_{\Lambda}(m)$ is nondecreasing for $m$ sufficiently large, say $m \geq d$. Thus by (5), for $m=$ $n-\sum_{i=1}^{\infty} l_{i} \lambda_{i}$, which satisfies $m \geq n-k n_{0} \sim n$, we have

$$
\prod_{i=1}^{\infty} \lambda_{i}^{-l_{i}}\left\{\sum_{\substack{b_{1}, \ldots, b_{k} \\ m-\Sigma b_{i} \geq 0}} P_{\Lambda}\left(m-\sum_{i=1}^{k} b_{i}\right)-O\left(m^{k-1}\right)\right\}
$$




$$
\begin{aligned}
& \leq \sum_{\substack{c_{1}, \ldots, c_{k} \\
m-\Sigma c_{i} \lambda_{j_{i}} \geq 0}} P_{\Lambda}\left(m-\sum_{i=1}^{k} c_{i} \lambda_{j_{i}}\right) \\
& \leq \prod \lambda_{i}^{-l_{i}}\left\{\sum_{\substack{b_{1}, \ldots, b_{k} \\
m+\Sigma l_{i} \lambda_{i}-\Sigma b_{i} \geq 0}} P_{\Lambda}\left(m+\sum_{i=1}^{\infty} l_{i} \lambda_{i}-\sum_{i=1}^{k} b_{i}\right)+O\left(m^{k-1}\right)\right\},
\end{aligned}
$$

since there are $O\left(m^{k-1}\right)$ solutions of $0 \leq m-\sum_{i=1}^{k} b_{i} \leq d$ in positive integers $b_{1}, \ldots, b_{k}$. Here $\lambda_{j_{1}}, \ldots, \lambda_{j_{k}}$ are the elements $\lambda_{i}$ with multiplicity $l_{i}$. Clearly,

$$
\sum_{\substack{b_{1}, \ldots, b_{k} \\ m-\Sigma b_{i} \geq 0}} P_{\Lambda}\left(m-\sum_{i=1}^{k} b_{i}\right)=P_{\Lambda_{k}}(m)
$$

and

$$
\sum_{\substack{c_{1}, \ldots, c_{k} \\ m-\Sigma c_{i} \lambda_{j_{i}} \geq 0}} P_{\Lambda}\left(m-\sum_{i=1}^{k} c_{i} \lambda_{j_{i}}\right)=P_{\Lambda^{\prime}}(m),
$$

so we deduce that

$$
\text { (6) } \begin{aligned}
\prod_{i=1}^{\infty} \lambda_{i}^{-l_{i}} P_{\Lambda_{k}}(m)(1+o(1)) & \leq P_{\Lambda^{\prime}}(m) \\
& \leq \prod_{i=1}^{\infty} \lambda_{i}^{-l_{i}} P_{\Lambda_{k}}\left(m+\sum_{i=1}^{k} l_{i} \lambda_{i}\right)(1+o(1)),
\end{aligned}
$$

because $P_{\Lambda_{k}}(m) \geq P_{\Lambda}(m)$ and, by $(2), \log \left\{P_{\Lambda}(m) / m^{k-1}\right\} \sim(1+1 / \alpha)$ $\times M m^{\alpha /(\alpha+1)}$. It is easily seen that the theorems of Ingham et al. and Meinardus apply to $\Lambda_{k}$; for the former, $N(n)$ corresponding to $\Lambda$ becomes $N(n)+k$ for $n \geq 1$, and for the latter $D(s)$ and $g(\tau)$ corresponding to $\Lambda$ become $D(s)+k$ and $g(\tau)+k e^{-\tau}$. We obtain in particular

$$
\begin{aligned}
\frac{P_{\Lambda_{k}}\left(m+\sum l_{i} \lambda_{i}\right)}{P_{\Lambda_{k}}(m)} & =\frac{n^{-1 / 2+(a-1 / 2+k) /(\alpha+1)}}{\left(n-\sum l_{i} \lambda_{i}\right)^{-1 / 2+(a-1 / 2) /(\alpha+1)}} \\
& \times \exp \left\{\left(1+\alpha^{-1}\right) M\left[n^{\alpha /(\alpha+1)}-\left(n-\sum l_{i} \lambda_{i}\right)^{\alpha /(\alpha+1)}\right]\right\},
\end{aligned}
$$

and since

$$
n^{\alpha /(\alpha+1)}-\left(n-k n_{0}\right)^{\alpha /(\alpha+1)} \sim \frac{\alpha}{\alpha+1} \frac{k}{\omega(n)} \rightarrow 0,
$$

we see that

$$
P_{\Lambda_{k}}\left(m+\sum_{i=1}^{\infty} l_{i} \lambda_{i}\right)=P_{\Lambda_{k}}(m)(1+o(1))
$$


where $o(1)$ depends on the $l_{i}$ only through $k$. Now (4) follows from (6). Hence

$$
\sum_{\substack{\Sigma a_{i} \lambda_{i}=n \\ \text { partitions }}} \prod_{i=1}^{\infty} a_{i}^{\left(l_{i}\right)}=\prod_{i=1}^{\infty}\left(\frac{l_{i} !}{\lambda_{i}^{l_{i}}}\right) P_{\Lambda_{k}}(n)(1+o(1)),
$$

and

$$
\begin{aligned}
E\left(\prod_{i=1}^{\infty} a_{i}^{\left(l_{i}\right)}\right) & =\prod_{i=1}^{\infty}\left(\frac{l_{i} !}{\lambda_{i}^{l_{i}}}\right) \frac{P_{\Lambda_{k}}(n)}{P_{\Lambda}(n)}(1+o(1)) \\
& =\prod_{i=1}^{\infty}\left(\frac{l_{i} !}{\lambda_{i}^{l_{i}}}\right) M^{-k} n^{k /(1+\alpha)}(1+o(1))
\end{aligned}
$$

by the theorem of Ingham et al. or Meinardus, where $o(1)$ depends on the $l_{i}$ only through $k$.

We now prove by induction on $k$ that, provided $l_{i}=0$ for $i$ such that $\lambda_{i}>n_{0}$,

$$
E\left(\prod_{i=1}^{\infty} a_{i}^{l_{i}}\right)=E\left(\prod_{i=1}^{\infty} a_{i}^{\left(l_{i}\right)}\right)(1+o(1))
$$

as $n \rightarrow \infty$. Let

$$
R=E\left(\prod_{i=1}^{\infty} a_{i}^{\left(l_{i}\right)}\right)-E\left(\prod_{i=1}^{\infty} a_{i}^{l_{i}}\right)
$$

Then

$$
R=\sum_{d=1}^{k} \sum_{\substack{j_{1}, j_{2}, \ldots \\ 0 \leq j_{i} \leq l_{i} \\ \Sigma\left(l_{i}-j_{i}\right)=d}}\left(\prod_{i=1}^{\infty} c_{j_{i}}\right) E\left(\prod_{i=1}^{\infty} a_{i}^{j_{i}}\right),
$$

where $c_{j_{i}}$ is the coefficient of $r^{j_{i}}$ in the polynomial $r^{\left(l_{i}\right)}$. Observe that $\prod_{i=1}^{\infty} c_{j_{i}}$ is bounded in terms of $k$, for

$$
\left|c_{j_{i}}\right| \leq\left(\begin{array}{c}
l_{i}-1 \\
j_{i}-1
\end{array}\right)\left(l_{i}-1\right)^{j_{i}} \leq l_{i}^{2 j_{i}}
$$

for $j_{i} \geq 1$, and so

$$
\prod_{i=1}^{\infty}\left|c_{j_{i}}\right| \leq\left(\max l_{i}\right)^{2 k} \leq k^{2 k}
$$

The claim (8) is trivial if $k=0$, so we assume that $k>0$ and that (8) holds for all $j_{1}, j_{2}, \ldots$ with $\sum_{i=1}^{\infty} j_{i}<k, j_{i} \leq l_{i}$, when $l_{i}$ is replaced by $j_{i}$. Then by (7), (9) and the induction hypothesis,

$$
\left|R\left\{E\left(\prod a_{i}^{\left(l_{i}\right)}\right)\right\}^{-1}\right|
$$




$$
\begin{aligned}
& \leq A_{1} \sum_{d=1}^{k} \sum_{\substack{j_{1}, j_{2}, \ldots \\
j_{i} \leq l_{i} \\
\Sigma\left(l_{i}-j_{i}\right)=d}} E\left(\prod a_{i}^{j_{i}}\right) M^{k} n^{-k /(1+\alpha)} \prod_{i=1}^{\infty}\left(\lambda_{i}^{l_{i}} / l_{i} !\right) \\
& \leq A_{2} \sum_{d=1}^{k} M^{d} n^{-d /(1+\alpha)} \sum_{\substack{j_{1}, j_{2}, \ldots \\
j_{i} \leq l_{i} \\
\Sigma\left(l_{i}-j_{i}\right)=d}} \prod_{i=1}^{\infty}\left\{\frac{j_{i} !}{l_{i} !} \lambda_{i}^{l_{i}-j_{i}}\right\} \\
& \leq A_{3} \sum_{d=1}^{k} n^{-d /(1+\alpha)}\left\{\sum_{\substack{i \\
l_{i}>0}} \lambda_{i}\right\}^{d} \\
& \leq A_{3} \sum_{d=1}^{k} n^{-d /(1+\alpha)}\left\{k n^{1 /(1+\alpha)} / \omega(n)\right\}^{d}=O(1 / \omega(n)),
\end{aligned}
$$

where $A_{1}, A_{2}$ and $A_{3}$ depend only on $k$.

Hence if $l_{i}=0$ for $i$ with $\lambda_{i}>n_{0}$,

$$
E\left(\prod_{i=1}^{\infty} a_{i}^{l_{i}}\right)=\prod_{i=1}^{\infty}\left(l_{i} ! / \lambda_{i}^{l_{i}}\right) M^{-k} n^{k /(1+\alpha)}(1+o(1)),
$$

where, again, $o(1)$ depends on the $l_{i}$ only through $k$. We have

$$
\begin{aligned}
E\left\{\left(\sum_{i=1}^{\infty} a_{i} / n^{1 /(1+\alpha)}\right)^{k}\right\}= & E\left\{\sum_{\substack{l_{1}, l_{2}, \ldots \\
\Sigma l_{i}=k}}\left(\begin{array}{c}
k \\
l_{1}, l_{2}, \ldots
\end{array}\right) \prod_{i=1}^{\infty} a_{i}^{l_{i}} / n^{k /(1+\alpha)}\right\} \\
= & E\left\{\sum_{(0)}\left(\begin{array}{c}
k \\
l_{1}, l_{2}, \ldots
\end{array}\right) \prod_{i=1}^{\infty} a_{i}^{l_{i}} / n^{k /(1+\alpha)}\right\} \\
& +\sum_{d=1}^{k} E\left\{\sum_{(d)}\left(\begin{array}{c}
k \\
l_{1}, l_{2}, \ldots
\end{array}\right) \prod_{i=1}^{\infty} a_{i}^{l_{i}} / n^{k /(1+\alpha)}\right\} \\
= & E_{1}+E_{2},
\end{aligned}
$$

say, where $\sum_{(d)}$ denotes a sum over all $l_{1}, l_{2}, \ldots$ such that $\sum l_{i}=k$ and

$$
\sum_{\substack{i \\ \lambda_{i}>n_{0}}} l_{i}=d
$$

We have by (10) that

$$
E_{1}=k ! M^{-k} \sum_{(0)} \prod_{i=1}^{\infty} \lambda_{i}^{-l_{i}}(1+o(1)),
$$


and since

$$
\begin{aligned}
& E\left\{\sum_{(0)} \prod_{i=1}^{\infty} a_{i}^{l_{i}}\right\} \\
& \leq E\left\{\sum_{\substack{l_{1}, l_{2}, \ldots \\
\Sigma l_{i}=k-d \\
l_{i}=0 \text { if } \lambda_{i}>n_{0}}} \prod_{i=1}^{\infty} a_{i}^{l_{i}}\right\}\left\{\max \sum_{\substack{i \\
\lambda_{i}>n_{0}}} a_{i}\right\}^{d} \\
& \leq \sum_{\substack{l_{1}, l_{2}, \ldots \\
\Sigma l_{i}=k-d \\
l_{i}=0 \text { if } \lambda_{i}>n_{0}}} \prod_{i=1}^{\infty}\left(l_{i} ! / \lambda_{i}^{l_{i}}\right) M^{-(k-d)} n^{(k-d) /(1+\alpha)}(1+o(1))\left\{n^{\alpha /(\alpha+1)} \omega(n)\right\}^{d} \\
& \leq k !\left(\sum_{\substack{i \\
\lambda_{i} \leq 0}} 1 / \lambda_{i}\right)^{k-d} M^{-(k-d)} n^{(k-d(1-\alpha)) /(1+\alpha)} \omega(n)^{d}(1+o(1)),
\end{aligned}
$$

we have the bound

$$
\begin{aligned}
\text { (12) } \quad E_{2} \leq(k !)^{2} \sum_{d=1}^{k}\left(\sum_{\substack{i \\
\lambda_{i} \leq n_{0}}} 1 / \lambda_{i}\right)^{k-d} \\
\quad \times M^{-(k-d)} n^{-d(1-\alpha) /(1+\alpha)} \omega(n)^{d}(1+o(1)) .
\end{aligned}
$$

If $\alpha<1$, and so $\sum 1 / \lambda_{i}$ converges, we have $E_{2} \rightarrow 0$ as $n \rightarrow \infty$ provided we choose $\omega(n)$ so that $\omega(n)=o\left(n^{(1-\alpha) /(1+\alpha)}\right)$. Hence by (11),

$$
\lim _{n \rightarrow \infty} E\left\{\left(M \sum_{i=1}^{\infty} a_{i} / n^{1 /(1+\alpha)}\right)^{k}\right\}=k ! \sum_{\substack{l_{1}, l_{2}, \ldots . \\ \Sigma l_{i}=k}} \prod_{i=1}^{\infty} \lambda_{i}^{-l_{i}}=k ! r_{k},
$$

where $r_{k}$ is the coefficient of $x^{k}$ in the power-series

$$
g(x)=\prod_{i=1}^{\infty}\left(1-x / \lambda_{i}\right)^{-1},
$$

and so if $M \sum a_{i} / n^{1 /(1+\alpha)}$ converges in distribution, $g(x)$ is the moment generating function of the limit distribution. Since $\left(1-x / \lambda_{i}\right)^{-1}$ is the moment generating function associated with the distribution function

$$
1-e^{-\lambda_{i} x}, \quad x \geq 0,
$$

the product

$$
\prod_{i=1}^{m}\left(1-x / \lambda_{i}\right)^{-1}
$$


is the moment generating function of the sum of $m$ independent random variables with the distributions (13) for $1 \leq i \leq m$. Since the product converges to a function continuous at zero, $g(x)$ is a moment generating function for a proper distribution function and $M \sum a_{i} / n^{1 /(1+\alpha)}$ converges to this distribution. The necessary probability theory can be found in [9], Chapter 6, especially Theorems 6.2 and 6.16. If the $\lambda_{i}$ are distinct, then the partial fractions representation,

$$
\prod_{i=1}^{m}\left(1-x / \lambda_{i}\right)^{-1}=\sum_{i=1}^{m}\left\{\prod_{\substack{j=1 \\ j \neq i}}^{m}\left(1-\lambda_{i} / \lambda_{j}\right)^{-1}\right\}\left(1-x / \lambda_{i}\right)^{-1}
$$

shows that the distribution associated with (14) is

$$
1-\sum_{i=1}^{m} \prod_{\substack{j=1 \\ j \neq i}}^{m}\left(1-\lambda_{i} / \lambda_{j}\right)^{-1} e^{-\lambda_{i} x}, \quad x \geq 0 .
$$

This converges to

$$
1-\sum_{i=1}^{\infty} \prod_{\substack{j=1 \\ j \neq i}}^{\infty}\left(1-\lambda_{i} / \lambda_{j}\right)^{-1} e^{-\lambda_{i} x}
$$

for $x>0$, provided

$$
\limsup _{i \rightarrow \infty} \prod_{\substack{j=1 \\ j \neq i}}^{\infty}\left|1-\lambda_{i} / \lambda_{j}\right|^{-1 / \lambda_{i}} \leq 1
$$

and so the second part of the theorem is established.

If $\alpha=1$, then by (11) for $k=1$,

$$
\lim _{n \rightarrow \infty} M E_{1}\left\{\sum_{\substack{i \\ \lambda_{i} \leq n_{0}}} 1 / \lambda_{i}\right\}^{-1}=1
$$

and by (12) for $k=1$,

$$
\lim _{n \rightarrow \infty} M E_{2}\left\{\sum_{\substack{i \\ \lambda_{i} \leq n_{0}}} 1 / \lambda_{i}\right\}^{-1} \leq \lim _{n \rightarrow \infty}\left(M\left\{\sum_{\substack{i \\ \lambda_{i} \leq n_{0}}} 1 / \lambda_{i}\right\}^{-1} \omega(n)\right)=0,
$$

provided $\omega(n)$ grows sufficiently slowly that

$$
\lim _{n \rightarrow \infty}\left\{\omega(n) / \sum_{\substack{i \\ \lambda_{i} \leq n^{1 / 2} / \omega(n)}} 1 / \lambda_{i}\right\}=0 .
$$


Similarly, for $k=2$,

$$
\begin{aligned}
\lim _{n \rightarrow \infty} M^{2} E_{1}\left\{\sum_{\substack{i \\
\lambda_{i} \leq n_{0}}} 1 / \lambda_{i}\right\}^{-2} & \\
= & \lim _{n \rightarrow \infty}\left\{2\left[\sum_{\substack{i \leq n_{0} \\
\lambda_{i} \leq n_{0}}} 1 / \lambda_{i}^{2}+\sum_{\substack{i<j \\
\lambda_{i}, \lambda_{j} \leq n_{0}}} 1 / \lambda_{i} \lambda_{j}\right] /\left(\sum_{\substack{i \\
\lambda_{i} \leq n_{0}}} 1 / \lambda_{i}\right)^{2}\right\} \\
& =\lim _{n \rightarrow \infty}\left\{\left[\left(\sum_{\substack{i \\
\lambda_{i} \leq n_{0}}} 1 / \lambda_{i}\right)^{2}+\sum_{\substack{i \leq n_{0} \\
\lambda_{i} \leq n_{0}}} 1 / \lambda_{i}^{2}\right] /\left(\sum_{\substack{i \\
\lambda_{i} \leq n_{0}}} 1 / \lambda_{i}\right)^{2}\right\}=1,
\end{aligned}
$$

and

$$
\lim _{n \rightarrow \infty} M^{2} E_{2}\left\{\sum_{\substack{i \\ \lambda_{i} \leq n_{0}}} 1 / \lambda_{i}\right\}^{-2}=\lim _{n \rightarrow \infty} O\left(\omega(n) / \sum_{\substack{i \\ \lambda_{i} \leq n_{0}}} 1 / \lambda_{i}\right)=0 .
$$

Hence, for

$$
Y_{n}=M\left\{\sum_{\substack{i \\ \lambda_{i} \leq n_{0}}} 1 / \lambda_{i}\right\}^{-1} \sum_{i=1}^{\infty} a_{i} / n^{1 / 2}
$$

we have

$$
\lim _{n \rightarrow \infty} E Y_{n}=1 \quad \text { and } \quad \lim _{n \rightarrow \infty} \operatorname{var} Y_{n}=0,
$$

and so $Y_{n}$ converges in distribution to one. We have thus shown that

$$
\sum_{\substack{i \\ \lambda_{i} \leq n_{0}}} 1 / \lambda_{i}
$$

is asymptotically independent of $\omega(n)$ provided $\omega(n)$ grows sufficiently slowly. It follows that for any $f(n)$ increasing to infinity and any $\omega_{1}(n)$ and $\omega_{2}(n)$ tending to infinity sufficiently slowly compared to $f(n)$,

$$
\sum_{\substack{i \\ \lambda_{i} \leq f(n) / \omega_{1}(n)}} 1 / \lambda_{i} \sim \sum_{\substack{i \\ \lambda_{i} \leq f(n) / \omega_{2}(n)}} 1 / \lambda_{i}
$$

If we take $f(n)=n^{1 / 2} \omega_{1}(n)$ and arrange that $\omega_{2}(n) / \omega_{1}(n) \rightarrow \infty$, we see that we may replace $n_{0}$ in (16) by $n^{1 / 2}$. When we note that

$$
M=\{A \Gamma(2) \zeta(2)\}^{1 / 2}=\left\{A \pi^{2} / 6\right\}^{1 / 2},
$$

we have the first part of the theorem.

ExAmples. (a) $\Lambda=\{1,2,3, \ldots\}=\mathbb{N}$. Meinardus's theorem applies with $D(s)=\zeta(s)$ and $g(\tau)=e^{-\tau} /\left(1-e^{-\tau}\right)$. Ingham's theorem applies with 
$R(n)=[n]-n$. In either case, we have $\alpha=A=1$ and

$$
\sum_{\substack{i \\ \lambda_{i} \leq n^{1 / 2}}} 1 / \lambda_{i} \sim(1 / 2) \log n,
$$

whence the first part of our theorem gives the result of Erdős and Lehner.

(b) $\Lambda$ a union of arithmetic progressions, $\Lambda=\left\{m \in \mathbb{N}: m \equiv b_{1}, b_{2}, \ldots\right.$, or $\left.b_{l} \bmod k\right\},\left(b_{1}, b_{2}, \ldots, b_{l}, k\right)=1$. Meinardus's theorem applies with

$$
D(s)=\sum_{i=1}^{l} k^{-s} \zeta\left(s, b_{i} / k\right)
$$

and

$$
g(\tau)=\sum_{i=1}^{l} e^{-b_{i} \tau} /\left(1-e^{-k \tau}\right) .
$$

Ingham's theorem applies with

$$
R(n)=l\{[n / k]-n / k\}+c_{n},
$$

where $c_{n}$ of the $b_{i}$ belong to congruence classes modulo $k$ with representatives in the interval $([n / k] k, n]$. In either case we have $\alpha=1, A=l / k$ and

$$
\sum_{\substack{i \\ \lambda_{i} \leq n^{1 / 2}}} 1 / \lambda_{i} \sim(l / 2 k) \log n,
$$

whence by the first part of our theorem,

$$
\pi(2 k / 3 l)^{1 / 2}\left(n^{1 / 2} \log n\right)^{-1} \sum_{i=1}^{\infty} a_{i}
$$

converges in distribution to one.

(c) $\Lambda$ the set of squares. Meinardus's theorem applies with $D(s)=\zeta(2 s)$ and $g(\tau)=\sum_{r=1}^{\infty} e^{-r^{2} \tau}$. One way to see that $g(\tau)$ satisfies the required condition is as follows. If, as Meinardus, we put $\tau=y+2 \pi i x$, where $y$ and $x$ are real, then for $y>0$,

$$
\begin{aligned}
g(y)-\operatorname{Re} g(\tau) & =\sum_{n=1}^{\infty} e^{-n^{2} y}\left(1-\cos \left(2 \pi n^{2} x\right)\right) \\
& \geq \sum_{\substack{n=1 \\
\cos \left(2 \pi n^{2} x\right) \leq 0}}^{\infty} e^{-n^{2} y} \geq \sum_{\substack{n=1 \\
\cos \left(2 \pi n^{2} x\right) \leq 0}}^{\left[\{\log 2 / y\}^{1 / 2}\right]} 1 / 2 .
\end{aligned}
$$

As was first shown by Weyl [10], if $x$ is irrational the sequence $\left(n^{2} x\right)_{n \geq 1}$ is uniformly distributed modulo one, and so, given $\eta>0$, the number of 
summands in $(17)$ is at least $\left(\frac{1}{2}-\eta\right)\{\log 2 / y\}^{1 / 2}$ for sufficiently small $y$. Hence for $x$ irrational,

$$
g(y)-\operatorname{Re} g(\tau) \geq \frac{1}{5}(\log 2)^{1 / 2} y^{-1 / 2}
$$

for small enough $y$. Finally, $g(\tau)$ is continuous for $y>0$, since in any half-plane $y \geq a>0$, the sum in the definition of $g(\tau)$ converges uniformly. Therefore (18) holds also for rational $x$, and we may take Meinardus's $\varepsilon$ to be $1 / 2$.

Ingham's theorem applies also, since we have $R(n)=\left[n^{1 / 2}\right]-n^{1 / 2}$ and

$$
\begin{aligned}
\lim _{u \rightarrow \infty}\left\{\int_{1}^{u} \frac{v^{1 / 2}-\left[v^{1 / 2}\right]}{v} d v-\frac{1}{2} \log u\right\} \\
\quad=\lim _{u \rightarrow \infty} \int_{1}^{u} \frac{v^{1 / 2}-\left[v^{1 / 2}\right]-\frac{1}{2}}{v} d v=\lim _{n \rightarrow \infty} \sum_{r=1}^{n} \int_{r^{2}}^{(r+1)^{2}} \frac{v^{1 / 2}-\left[v^{1 / 2}\right]-\frac{1}{2}}{v} d v \\
=\lim _{n \rightarrow \infty} \sum_{r=1}^{n}\{2-(2 r+1) \log (1+1 / r)\}=\lim _{n \rightarrow \infty} \sum_{r=1}^{n} O\left(1 / r^{2}\right)
\end{aligned}
$$

exists. We have $A=\alpha=1 / 2$. Our theorem now says that

$$
M \sum_{i=1}^{\infty} a_{i} / n^{2 / 3}
$$

converges in distribution to the distribution

$$
F(x)=1-\lim _{m \rightarrow \infty} \sum_{r=1}^{m}\left\{\prod_{\substack{j=1 \\ j \neq r}}^{m}\left(1-r^{2} / j^{2}\right)^{-1}\right\} e^{-r^{2} x},
$$

and since

$$
\begin{aligned}
\prod_{\substack{j=1 \\
j \neq r}}^{\infty}\left(1-r^{2} / j^{2}\right)^{-1} & =(-1)^{r-1} \prod_{j=1}^{r-1} \frac{j^{2}}{(r-j)(r+j)} \prod_{j=r+1}^{\infty} \frac{j^{2}}{(j-r)(j+r)} \\
& =(-1)^{r-1} \frac{(r-1) !^{2} r}{(2 r-1) !} \frac{(2 r) !}{r !^{2}}=(-1)^{r-1} \cdot 2,
\end{aligned}
$$

we have

$$
F(x)=\sum_{r=-\infty}^{\infty}(-1)^{r} e^{-r^{2} x}, \quad x>0 .
$$

By the result of Jacobi and Gauss, given for example on page 23 of [1],

$$
F(x)=\prod_{m=1}^{\infty} \frac{1-e^{-m x}}{1+e^{-m x}}, \quad x \geq 0 .
$$


Hence if the factor $\log n$ is removed from his original question, Erdős's distribution function $f(c)$ satisfies

$$
f(c)=F(M c),
$$

where, since $\Gamma\left(\frac{3}{2}\right)=\frac{1}{2} \sqrt{\pi}$,

$$
M=\left\{\frac{1}{4} \sqrt{\pi} \zeta\left(\frac{3}{2}\right)\right\}^{2 / 3} .
$$

In particular the distribution given by $f(c)$ has mean

$$
\frac{1}{6} \pi^{5 / 3}\left\{4 / \zeta\left(\frac{3}{2}\right)\right\}^{2 / 3}=1.49 \ldots
$$

and variance

$$
\frac{1}{90} \pi^{10 / 3}\left\{4 / \zeta\left(\frac{3}{2}\right)\right\}^{4 / 3}=0.89 \ldots
$$

(d) $\Lambda$ the set of $k$-th powers, $k \geq 2$. By similar reasoning as in (c), the theorems of Meinardus and Ingham apply. Note that for $k>2$,

$$
\begin{aligned}
-r^{-k} \sum_{\substack{j=1 \\
j \neq r}}^{\infty} \log \left|1-r^{k} / j^{k}\right| & =r^{-k} \sum_{j=1}^{r-1} \log \left(\frac{j^{k}}{r^{k}-j^{k}}\right)+r^{-k} \sum_{j=r+1}^{\infty} \log \left(\frac{j^{k}}{j^{k}-r^{k}}\right) \\
& \leq r^{-(k-1)} k \log r+r^{-k} \sum_{j=r+1}^{\infty}\left(\frac{r^{k}}{j^{k}-r^{k}}\right),
\end{aligned}
$$

and for $j>r, j^{k-1}(j-1)>r^{k}$, whence

$$
-r^{-k} \sum_{\substack{j=1 \\ j \neq r}}^{\infty} \log \left|1-r^{k} / j^{k}\right| \leq r^{-(k-1)} k \log r+\sum_{j=r+1}^{\infty} 1 / j^{k-1} \rightarrow 0
$$

as $r \rightarrow \infty$. Hence

$$
M \sum_{i=1}^{\infty} a_{i} / n^{k /(k+1)}
$$

has a limit distribution of

$$
1-\sum_{r=1}^{\infty}\left\{\prod_{\substack{j=1 \\ j \neq r}}^{\infty}\left(1-r^{k} / j^{k}\right)^{-1}\right\} e^{-r^{k} x}, \quad x>0,
$$

where

$$
M=\left\{k^{-1} \Gamma(1+1 / k) \zeta(1+1 / k)\right\}^{k /(k+1)} .
$$

By the Weierstrass product form for the reciprocal of the gamma function,

$$
\prod_{j=1}^{\infty}\left(1-x^{k} / j^{k}\right)=\prod_{\substack{\varrho \\ \varrho^{k}=1}} 1 / \Gamma(1-\varrho x),
$$


and so

$$
\begin{aligned}
\prod_{\substack{j=1 \\
j \neq r}}^{\infty}\left(1-r^{k} / j^{k}\right) & =\lim _{x \rightarrow r}\left\{\prod_{\substack{\varrho \\
\varrho^{k}=1}} \Gamma(1-\varrho x)\left(1-x^{k} / r^{k}\right)\right\} \\
& =k \prod_{\substack{\varrho \\
\varrho^{k}=1, \varrho \neq 1}} \Gamma(1-\varrho r) \lim _{x \rightarrow r}(1-x / r) \Gamma(1-x) \\
& =k \prod_{\substack{\varrho \\
\varrho^{k}=1, \varrho \neq 1}} \Gamma(1-\varrho r) \lim _{x \rightarrow r} \frac{(1-x / r) \pi}{\Gamma(x) \sin (\pi x)} \\
& =\frac{(-1)^{r-1}}{r !} k \prod_{\substack{\varrho \\
\varrho^{k}=1, \varrho \neq 1}} \Gamma(1-\varrho r) .
\end{aligned}
$$

In particular, for $k=4$ we have the distribution function

$$
1-\sum_{r=1}^{\infty}(-1)^{r-1} \frac{4 \pi r}{\sinh (\pi r)} e^{-r^{4} x} .
$$

We remark that (15) leads to the identity

$$
\prod_{r=1}^{\infty}\left(1-x / r^{4}\right)^{-1}=\sum_{r=1}^{\infty}(-1)^{r-1} \frac{4 \pi r}{\sinh (\pi r)}\left(1-x / r^{4}\right)^{-1}
$$

when $k=4$, and the identity

$$
\prod_{r=1}^{\infty}\left(1-x / r^{2}\right)^{-1}=\lim _{z \rightarrow 1^{-}} 2 \sum_{r=1}^{\infty}(-1)^{r-1} z^{r}\left(1-x / r^{2}\right)^{-1}
$$

when $k=2$. This last identity shows that $\zeta(2 n)$ is a rational multiple of $\zeta(2)^{n}$. As an example, comparison of the coefficients of $x^{2}$ on either side gives

$$
\sum_{r=1}^{\infty} 1 / r^{4}+\sum_{1 \leq r<s<\infty} 1 / r^{2} s^{2}=2 \sum_{r=1}^{\infty}(-1)^{r-1} \cdot 1 / r^{4},
$$

which is equivalent to $\frac{1}{2} \zeta(2)^{2}+\frac{1}{2} \zeta(4)=2\left(1-2 / 2^{4}\right) \zeta(4)$, whence $\zeta(4)=$ $\frac{2}{5} \zeta(2)^{2}$.

\section{References}

[1] G. E. Andrews, The Theory of Partitions, Addison-Wesley, 1976.

[2] F. C. Auluck and C. B. Haselgrove, On Ingham's Tauberian theorem for partitions, Proc. Cambridge Philos. Soc. 48 (1952), 566-570. 
[3] P. Bateman and P. Erdős, Monotonicity of partition functions, Mathematika 3 (1956), 1-14.

[4] P. Erdös and J. Lehner, The distribution of the number of summands in the partitions of a positive integer, Duke Math. J. 8 (1941), 335-345.

[5] P. Erdős and P. Turán, On some general problems in the theory of partitions, Acta Arith. 18 (1971), 53-62.

[6] A. E. Ingham, A Tauberian theorem for partitions, Ann. of Math. (2) 42 (1941), 1075-1090.

[7] J. H. Loxton and H.-F. Yeung, Common summands in partitions, Acta Arith. 60 (1992), 307-320.

[8] G. Meinardus, Asymptotische Aussagen über Partitionen, Math. Z. 59 (1954), 388-398.

[9] P. A. P. Moran, An Introduction to Probability Theory, Clarendon Press, 1968.

[10] H. Weyl, Über die Gleichverteilung von Zahlen mod. Eins, Math. Ann. 77 (1916), $315-352$.

MATHEMATICS DEPARTMENT

NOTTINGHAM UNIVERSITY

UNIVERSITY PARK

NOTTINGHAM NG7 2RD, U.K. 